\title{
Minimal inframammary incision for breast augmentation
}

\author{
Nabil Fanous MD FRCSC ${ }^{1,2}$, Caroline Tawilé MD FRCSC ${ }^{3}$, Valérie J Brousseau MDCM ${ }^{1}$
}

\begin{abstract}
N Fanous, C Tawilé, VJ Brousseau. Minimal inframammary incision for breast augmentation. Can J Plast Surg 2008;16(1):14-17.
\end{abstract}

The inframammary approach in breast augmentation, still the most popular technique among plastic surgeons, has always been hampered by the undesirable appearance of its scar. The present paper describes a modified approach to inframammary augmentation with salinefilled prostheses. This approach uses a very short incision, thus resulting in a much less noticeable scar. The surgical technique is easy to learn, simple to execute, does not necessitate any special equipment and gives consistent results. Decreasing the scar length to an absolute minimum ensures higher patient and surgeon satisfaction.

\section{Incision inframammaire minimale en vue d'une augmentation mammaire}

La voie d'abord inframammaire en vue d'une augmentation mammaire, la technique encore préférée des chirurgiens plasticiens, a toujours fait problème en raison de l'apparence indésirable de la cicatrice. Le présent article fait état d'une modification de la voie d'abord en vue d'une augmentation mammaire réalisée à l'aide de prothèses remplies de solution saline. Cette voie d'abord n'exige qu'une très courte incision, d'où cicatrice très discrète. La technique chirurgicale est facile à apprendre et à réaliser, ne nécessite pas de matériel particulier et donne des résultats constants. Ainsi, le raccourcissement de la cicatrice à une longueur minimale ne peut que plaire aux patientes et aux chirurgiens.

\section{Key Words: Breast augmentation; Incision; Inframammary; Short}

$\mathrm{M}$ ammary augmentation may be performed using the inframammary, periareolar, transaxillary or transumbilical approach (1-9). The surgeon's choice of approach is usually based on numerous factors such as the visibility of the incision, the surgeon's experience, the perceived surgical comfort level, the documented risks, the length of surgery, the length of recovery, the combination of the augmentation with other breast surgeries (such as mastopexies), the market tendencies and the media hypes. Of all these factors, the noticeability of the incision seems to be the main reason why surgeons opt for the periareolar, transaxillary or transumbilical approaches. Indeed, these approaches allow for the incisions to be more easily hidden or camouflaged.

Interestingly, the inframammary approach still remains the most common one practiced by plastic surgeons, and this in spite of its perceived disadvantage: the visibility of the scar. The persisting popularity of this approach is due to its many advantages: it is easy to learn; it offers a direct access to the submuscular, subpectoral or subglandular planes; the dissection may be precisely limited to the desired pocket boundaries without the need to dissect an access tract between the entry site and the actual pocket (as is the case in the three other approaches); it does not necessitate the purchase of expensive surgical equipment such as endoscopes; and it allows the use of dependent drains if the surgeon's technique includes them (1).

The present article describes an inframammary approach to mammary augmentation with saline-filled implants, using an incision that is shorter by approximately $50 \%$ than the one commonly performed. The resulting smaller scar is greatly appreciated by patients, thereby raising their satisfaction rate, as well as that of the surgeon.

\section{SURGICAL TECHNIQUE \\ The preoperative markings}

The existing inframammary crease is marked. The location of the new inframammary crease is then drawn at a slightly lower level, depending on the projected implant volume and the present position of the areola and nipple. Our suggested inframammary incision measures only $1.7 \mathrm{~cm}$. It is placed along the future inframammary crease and is centred on the meridian of the breast. The pocket to be dissected is also precisely drawn on the skin.

\section{The infiltration}

Figure 1 describes the consecutive steps of the 'minimal inframammary incision' augmentation technique. The incision site is infiltrated with $3 \mathrm{~mL}$ of xylocaine $1 \%$ with adrenaline $1: 1,000,000$. First, a small stab incision of approximately $3 \mathrm{~mm}$ in length is made in the centre of the future incision to allow the introduction of an infiltration cannula into the submuscular, subpectoral or subglandural space, depending on the plane chosen by the surgeon. Approximately $150 \mathrm{~mL}$ of tumescent solution, similar to the solution used in liposuction, is then injected to initiate pocket elevation and promote hemostasis (Figure 1A).

\section{The dissection}

Using a number 15 blade, the incision is then extended to its full length following the preoperative markings $(1.7 \mathrm{~cm}$ preoperatively). Blunt dissection is used to fashion the pocket,

${ }^{1}$ Institute of Cosmetic Surgery, Westmount, Montréal; ${ }^{2}$ Department of Surgery, Centre Hospitalier de Lachine; ${ }^{3}$ Department of Plastic Surgery, Hôpital Charlemagne, Montréal, Quebec

Correspondence: Dr Nabil Fanous, 1 Westmount Square, suite 1380, Montreal, Quebec H32 2P9. Telephone 514-935-9906,

fax 514-935-6482, e-mail cosmeticsurgery123@videotron.ca 

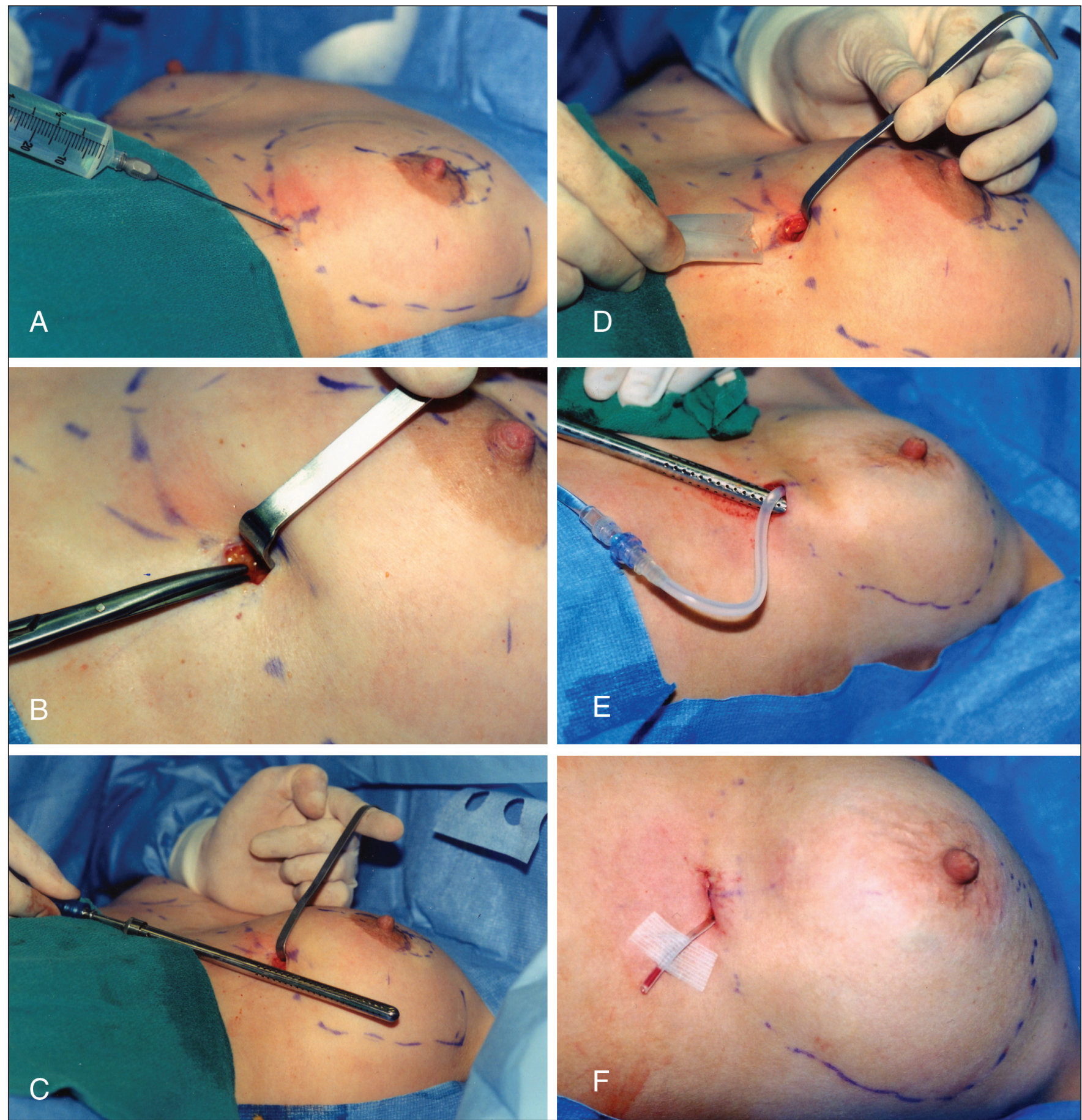

Figure 1) Consecutive steps of the 'minimal incision' inframammary augmentation technique. A Injection of tumescent solution through a 3 mm stab incision; B Dissection to penetrate into the muscular plane made up of the serratus anterior, the external oblique and the pectoralis major muscles; $\mathrm{C}$ Introduction of a metallic abdominal suction instrument (the 'metal finger') for pocket dissection and palpation; D Introduction of the folded implant using a thin, narrow, right-angled retractor; E Final dissection adjustments of the remaining tight or irregular areas of the pocket are performed with the metal finger, after the insertion and inflation of the implant; $\mathbf{F}$ Wound closure is completed, with a small drain left at the lateral aspect of the wound

whether it is submuscular, subpectoral or subglandular. We use a completely submuscular pocket under three muscles: the serratus anterior, the external oblique and the pectoralis major. This dissection is started using Metz scissors to form a small submuscular 'vestibule' (Figure 1B). Further dissection is then extended to lift the central part of the pocket using a metallic abdominal suction instrument, a commonly used instrument in general surgery (Figure 1C). Because we use it as a substitute to finger dissection during the procedure, we have nicknamed it the 'metal finger'. Peripheral dissection of the pocket is made with a Dingman mammary dissector. Then, for a final assessment of the pocket shape and size, the metal finger is again introduced and used to palpate the contour of the cavity and to complete any extra muscle dissection that is required. 


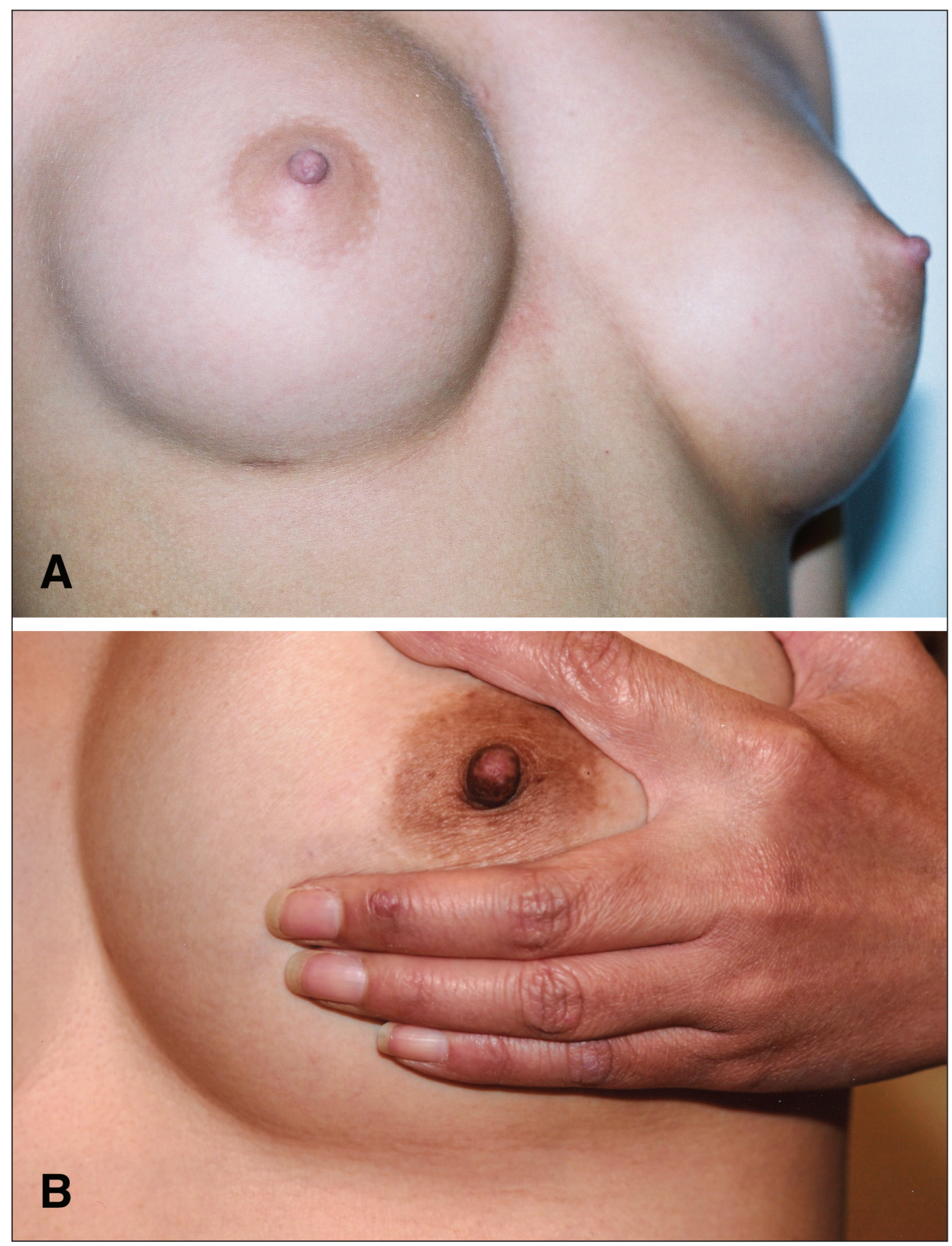

Figure 2) Two close-ups of minimal inframammary incisions at seven months (A) and 72 months (B) postoperatively

Depending on the surgeon's preference, a sterile implant sizer may be used at this point to determine the proper implant size selection. The same dissection technique is then performed on the opposite side. Hemostasis, if needed, is obtained with fine cautery. According to the surgeon's preference, the release of the resistant inferomedial or inferolateral muscle fibres may be achieved by blunt muscle stretching with the metal finger (our preferred choice) or by sectioning the tight muscle fibres with sharp dissection.

\section{Implant introduction}

Pocket irrigation with the surgeon's choice of solution is then performed (we use diluted bacitracin solution). The selected implant (we use the Mentor [Canada] smooth round implant) is then prepared and folded on itself like a tight sausage. A narrow (7 $\mathrm{mm}$ to $10 \mathrm{~mm}$ wide) retractor is used to maintain the incision, through which the implant is slowly introduced (Figure 1D). The implant is then inflated with the appropriate saline volume in the usual manner. Once the implant is inflated, the metal finger is rolled gently over its surface from right to left to push the valve in. If any segment of the pocket, regardless of its distance from the incision, needs additional dissection, the metal finger is again used, easily and efficiently, to do so (Figure 1E).

\section{Incision closure}

The incision is closed in the usual manner in two layers (Figure 1F). We prefer to leave a very narrow dependent drain through the incision for $24 \mathrm{~h}$ (1).

\section{Postoperative findings}

The postoperative findings of this approach are no different than those of the usual inframammary approach, except for the length of the scar. Interestingly, we noticed that the $1.7 \mathrm{~cm}$ scar shrinks by almost $2 \mathrm{~mm}$ with healing, reaching approximately $1.5 \mathrm{~cm}$ one year postoperatively. Scarring is minimal postoperatively (Figure 2).

\section{DISCUSSION}

The main contribution of the approach discussed above is the significant shortening of the inframammary incision used for saline-filled breast augmentation. According to surgeons and textbooks, the classical length of this incision varies between $3 \mathrm{~cm}$ and $5 \mathrm{~cm}$ (an average of $4.25 \mathrm{~cm})(2-5)$. Our technique suggests an incision of $1.7 \mathrm{~cm}$, which is approximately $50 \%$ shorter than the classical incision. Although the reduction of the incision from $3 \mathrm{~cm}, 4 \mathrm{~cm}$ or $5 \mathrm{~cm}$ to $1.7 \mathrm{~cm}$ may seem relatively insignificant, the difference is most impressive clinically to patients and surgeons. The usual $3 \mathrm{~cm}$ to $5 \mathrm{~cm}$ classical incision was required mostly for two reasons: to allow the use of one or more fingers, and occasionally part of the hand, for close and far dissection of the pocket; and to accommodate a variety of 
relatively large retractors used to introduce the implant. Our approach allows for a significantly smaller incision by substituting the fingers and hand with the metal finger, and the regular retractors with a very thin one.

The metal finger is the metal abdominal suction instrument readily available in any operating room. This instrument, which has approximately the diameter of a small finger, is most useful during this approach of augmentation mammoplasty. It is blunt, narrow and light, yet extremely resilient and powerful. When used with a gentle touch, it gives the surgeon an accurate feel for the pocket boundaries and can detect areas of incomplete dissection. When used with varying strength, it can dissect lightly or mightily, with high precision. It allows for careful and precise dissection for even a few millimeters at a time, under total control, and this regardless of whether the area is close to the incision or distant from it. It is stronger, more accurate, and can reach further into the pocket than a surgeon's hand, while requiring a much smaller incision and less physical force. The metal finger may also be used as a suction device, on and off, while using it as a dissector, which gives it further practicality.

The second important instrument that we use is a thin, narrow ( $7 \mathrm{~mm}$ to $10 \mathrm{~mm}$ wide) angled retractor. Any retractor with similar specifications may be used. The longer arm of this retractor, which is approximately $4 \mathrm{~cm}$ long, is introduced through the upper border of the incision, to lift the superficial aspect of the pocket and allow swift introduction of the implant.

It is important to take note that the short incision technique presented here only alters the length of the incision, the way the pocket is dissected and the way that the implant is introduced; however, it does not influence other steps of the augmentation mammoplasty procedure, such as the selected plane of dissection (submuscular, subpectoral or subglandular), the preferred shape and texture of the implant, the choice of the pocket irrigation solution, or the type of incision closure, etc. Therefore, using this technique only decreases the resultant scar length and visibility. However, it should not affect the results or complication rates of the procedure.

This technique has some limitations. It can only be performed with inflatable saline implants, because only an empty and folded implant can penetrate through such a small incision. There is also a theoretical possibility that a persistent deep intrapocket bleeder may require the introduction of an endoscope to visualize it, or simply the enlargement of the incision to reach it, but the authors have yet to face such a situation after having performed over 700 procedures.

\section{CONCLUSION}

Although the inframammary approach to breast augmentation remains very popular, the length and visibility of its associated scar continue to be the main drawback of this technique.

The present article describes a technique that significantly reduces the length of the usual inframammary incision, hence allowing for increased patient and surgeon satisfaction.

ACKNOWLEDGEMENTS: The authors express their thanks to Maria Iancu, administrative assistant, for the typing of the manuscript; to Minerva Khalife for her photographic contribution; to Barbara Armbruster, MA, for the editing, and to Amanda Fanous, McGill University Medical School, for the medical literature search and for organizing the manuscript.

Disclosure of potential conflict of interest: None of the authors have any potential conflict of interest to declare.

\section{REFERENCES}

1. Fanous N, Salem I, Tawile C, Bassas AE. Absence of capsular contracture in 319 consecutive augmentation mammaplasties: Dependent drains as a possible factor. Can J Plast Surg 2004;12:193-7.

2. Bostwick J III. Standard subpectoral inframammary augmentation. In: Bostwich J III, ed. Plastic and Reconstructive Breast Surgery, Vol 1. St Louis: Quality Medical Publishing Inc, 2000:331.

3. Bohmert H, Gabka CH. Complications in augmentation mammoplasty. In: Bohmert H, Gabka CH, eds. Plastic and Reconstructive Surgery of the Breast: A Surgical Atlas. New York: Thieme Medical Publishers, 1997;19.

4. Spear S. The inframammary approach to augmentation mammoplasty. In: Spear S, ed. Surgery of the Breast: Principles and Art. Philadelphia: Lippincott-Raven Publishers, 1998;862.

5. Going J. Augmentation mammaplasty. In: McKissock PK, ed. Color Atlas of Mammaplasty. New York: Thieme Medical Publishers, 1991:119.

6. Niechajev I. Mammary augmentation by cohesive silicone gel implants with anatomic shape: Technical considerations. Aesthetic Plast Surg 2001;25:397-403.

7. Graf RM, Bernards A, Rippel R, Araujo LR, Damasio RC, Auersvald A. Subfascial breast implant: A new procedure. Plast Reconstr Surg 2003;111:904-8.

8. Chajchir A, Benzaquen I, Spagnolo N, Lusicic N. Endoscopic augmentation mastoplasty. Aesthetic Plast Surg 1994;18:377-82.

9. Hidalgo DA. Breast augmentation: Choosing the optimal incision, implant, and pocket plane. Plast Reconstr Surg 2000;105:2202-16; discussion 2217-8. 